\title{
Particle Physics in Ice with IceCube DeepCore
}

\author{
Tyce DeYoung \\ for the IceCube collaboration \\ Department of Physics, Pennsylvania State University, University Park, PA 16802, U.S.A. \\ E-mail: deyoung@psu.edu
}

\begin{abstract}
The IceCube Neutrino Observatory is the world's largest high energy neutrino telescope, using the Antarctic ice cap as a Cherenkov detector medium. DeepCore, the low energy extension to IceCube, is an infill array with a fiducial volume of around 30 MTon in the deepest, clearest ice, aiming for an energy threshold as low as $10 \mathrm{GeV}$ and extending IceCube's sensitivity to indirect dark matter searches and atmospheric neutrino oscillation physics. We will discuss the analysis of the first year of DeepCore data, as well as ideas for a further extension of the particle physics program in the ice with a future PINGU detector.
\end{abstract}

Keywords: astroparticle physics; neutrino oscillations; dark matter

\section{Introduction}

The IceCube neutrino telescope, now fully operational at depths of 1450-2450 m below the surface of the Antarctic ice cap, was designed to detect high energy neutrinos from astrophysical accelerators of cosmic rays. Although the energy threshold of a large volume neutrino detector is not a sharp function, the original IceCube design focused on efficiency for neutrinos at $\mathrm{TeV}$ energies and above. Recently, the IceCube collaboration decided to augment the response of the detector at lower energies with the addition of DeepCore, a fully contained subarray aimed at improving the sensitivity of IceCube to neutrinos with energies in the range of 10 's of $\mathrm{GeV}$ to a few hundred GeV. This energy range is of interest for several topics related to particle physics, including measurements of neutrino oscillations and searches for neutrinos produced in the annihilation or decay of dark matter.

DeepCore consists of an additional eight strings of photosensors (Digital Optical Modules, or DOMs) comprising 10" Hamamatsu photomultiplier tubes and associated data acquisition electronics housed in standard IceCube glass pressure vessels. For most of the DeepCore DOMs, the standard IceCube R7081 PMTs were replaced with 7081MOD PMTs with Hamamatsu's new super-bialkali photocathode. These PMTs provide approximately $35 \%$ higher quantum efficiency (averaged over the detected Cherenkov spectrum) than the stan- dard bialkali PMTs.

Sited at the bottom center of the IceCube array, DeepCore benefits from the high optical quality of the ice at depths of 2100-2450 m, with an attenuation length of approximately $50 \mathrm{~m}$ in the blue wavelengths at which most Cherenkov photons are detected in ice. DeepCore also benefits from the ability of the standard IceCube sensors to detect atmospheric muons penetrating the ice from cosmic ray air showers above the detector, allowing substantial reduction in the background rate by vetoing events where traces of penetrating muons are seen. Each DeepCore string bears 50 DOMs in the fiducial region, with an additional 10 DOMs deployed at shallower depths to improve the vetoing efficiency for steeply vertical muons. In addition to the new DeepCore strings, the DeepCore fiducial volume for analysis includes 12 standard IceCube strings, chosen so that the fiducial region is shielded on all sides by a veto region consisting of three rows of standard IceCube strings, as shown in Fig. 1]

The random noise rate of IceCube DOMs is quite low (around $500 \mathrm{~Hz}$, on average) due to the low temperatures and radiopurity of the ice cap. This permits DeepCore to be operated with a very low trigger threshold, demanding that 3 DOMs within the DeepCore fiducial region detect light in "local coincidence" within a period of no more than $2500 \mathrm{~ns}$. The local coincidence criterion counts DOMs as being hit (i.e., having detected light) only if one of the four neighboring DOMs on a 


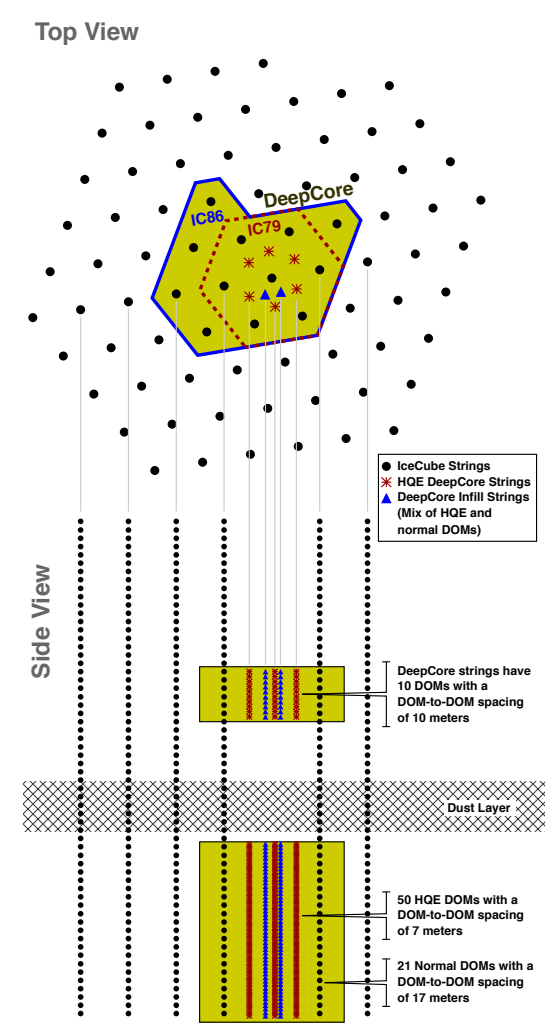

Figure 1: Schematic layout of DeepCore within IceCube. The shaded region indicates the fiducal volume of DeepCore, at the bottom center of IceCube, plus the extra veto cap of DOMs deployed at shallower depths to reinforce the veto against vertically-downgoing atmospheric muons. This schematic depicts both the DeepCore configuration used in 2010, when 79 IceCube strings were operational, and the final DeepCore layout and fiducial region used in the 2011 run.

string (two above and two below) also registers a hit within $\pm 1 \mu \mathrm{s}$. Most of the resulting $185 \mathrm{~Hz}$ of triggers are due to stray light from muons which simultaneously satisfy the main IceCube trigger condition of 8 DOMs hit in local coincidence within $5 \mu \mathrm{s}$, but the DeepCore trigger contributes an additional (exclusive) rate of around $10 \mathrm{~Hz}$.

The vast majority of the events which trigger DeepCore, irrespective of whether they also trigger IceCube, are due to either penetrating atmospheric muons or random coincidences of dark noise. Immediately after data acquisition, events triggering DeepCore are subjected to an online data rejection algorithm which calculates a characteristic time and location for the activity observed in the DeepCore fiducial region, as an initial estimate of the putative neutrino vertex. The estimated location is the average position of the hit DOMs, and the time

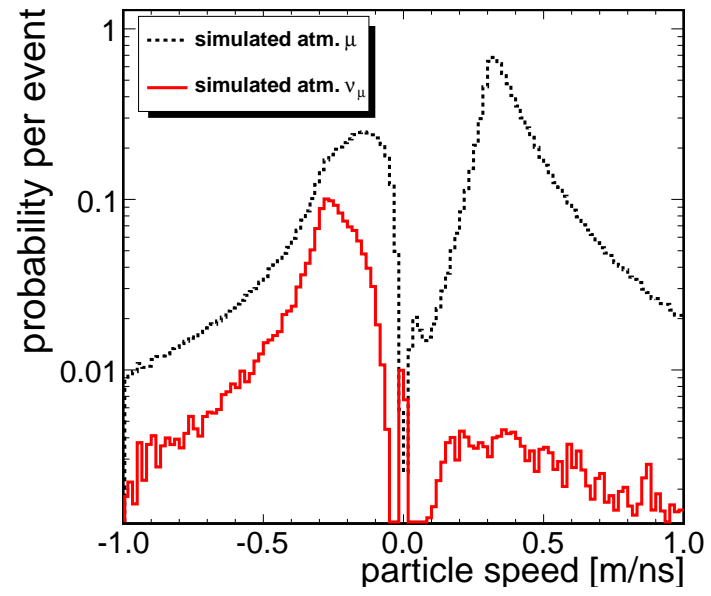

Figure 2: Distribution of probabilities of observing hits leading to a given inferred particle speed, for simulated atmospheric muons (dashed line) and atmospheric neutrinos (solid). Positive speeds indicate activity in the veto region prior to that in the DeepCore volume, and a peak around $c=0.3 \mathrm{~m} / \mathrm{ns}$ is visible for penetrating muons. The integral of each distribution corresponds to the mean number of hits observed in the veto region for the given class of events.

is determined by subtracting the time of flight $d n / c$ of an unscattered photon emitted from that location from the observed arrival time of the first photon to hit each DOM. After outliers due to dark noise or scattered light are removed, the average inferred emission time is used as the estimated time of the underlying physics event.

Based on this estimated time and location, every locally coincident hit recorded in the veto region prior to the vertex time is examined to determine whether it lies on the light cone connecting it with the estimated event vertex. The distributions of the inferred speed required to connect hits in the veto region to the DeepCore vertex, for both simulated atmospheric muons and simulated neutrinos, is shown in Fig. 2, positive speeds indicate hits occuring in the veto region prior to the DeepCore vertex time. If any hits are found with inferred speeds between +0.25 and $+0.4 \mathrm{~m} / \mathrm{ns}$, the event is rejected as being most likely due to an atmospheric muon. This algorithm reduces the event rate by more than two orders of magnitude, to $18 \mathrm{~Hz}$, while retaining over $99 \%$ of simulated triggered events due to neutrinos interacting within the fiducial volume. Additional background rejection criteria are applied offline, depending on the goals of each physics analysis making use of these data.

The effective volume of the DeepCore detector for detection low energy muon neutrinos, accounting for this online data filter, is shown in Fig. 3. It should be stressed that this effective volume curve does not in- 


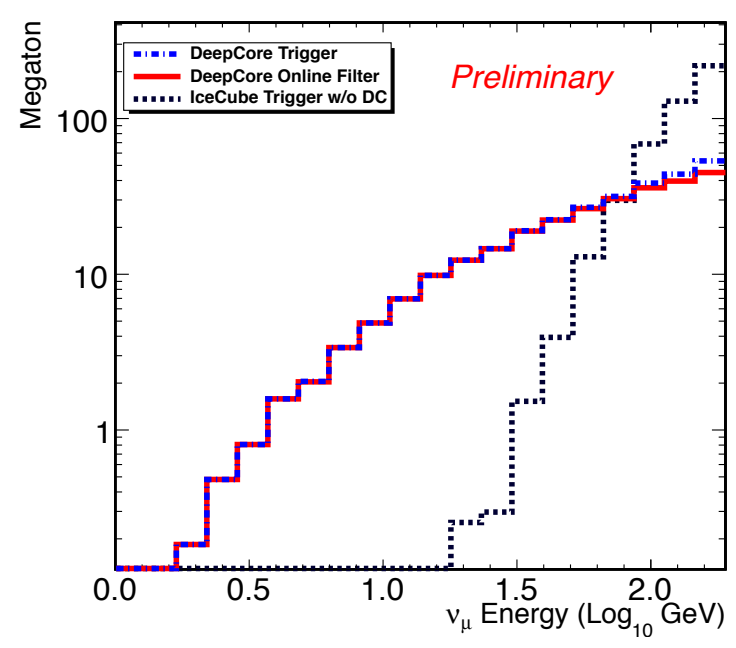

Figure 3: Effective volume of DeepCore for muon neutrinos at trigger level (solid) and after application of the online veto algorithm described in the text (dot-dashed line). The effective volume of IceCube as originally proposed is shown for comparison.

clude losses due to later background rejection or event quality criteria. The contribution of DeepCore to low energy analysis is evident in the fact that despite its relatively small geometric volume, around 3\% that of IceCube, the overall sample of neutrino events below $100 \mathrm{GeV}$ consists primarily of those detected by DeepCore. This energy range is of considerable interest for several topics in particle physics, including searches for dark matter and measurements of neutrino oscillations. While DeepCore does not have a sharp energy threshold, it retains around 7 megatons of effective volume at energies as low as $10 \mathrm{GeV}$. Further details regarding DeepCore's instrumentation and performance are available in Ref. [1].

\section{Observation of Neutrino-Induced Cascades}

Using the first year of data recorded with DeepCore, from May 2010 to April 2011, we have observed cascades induced by atmospheric neutrinos interacting in the DeepCore volume. These cascades include charged current (CC) interactions of electron neutrinos, as well as neutral current (NC) interactions of neutrinos of all flavors. (The background rejection criteria used in this analysis result in an energy threshold of around 40 $\mathrm{GeV}$, so only a negligible contribution from atmospheric muon neutrinos oscillating to tau is expected.) Previous searches for neutrino-induced cascades in AMANDA and IceCube [2, 3, 4, 5, 6] have focused on higher energies, to avoid the background of bremsstrahlung pro-

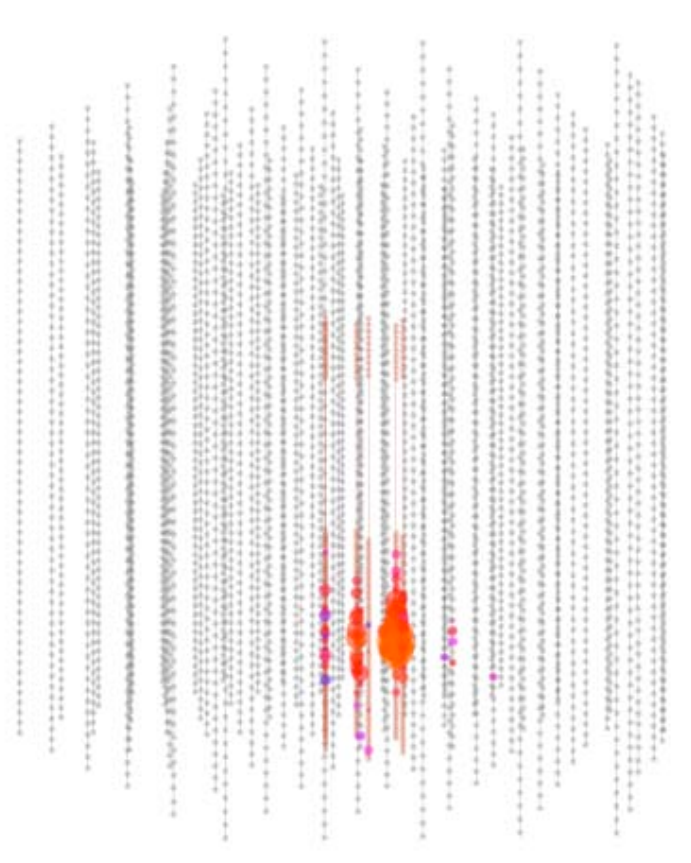

Figure 4: Candidate neutrino-induced cascade observed in DeepCore in the 2010 data run. Each black dot indicates a DOM. Colored dots represent DOMs that detected light during the event, with the size of the dot proportional to the amount of light detected. The color indicates the relative arrival time of the first photon detected by that DOM, running through the spectrum from red (earliest) to purple (latest).

duced by atmospheric muons. In this analysis, we instead rely on the active veto provided by IceCube to reduce the background of penetrating muons, and exploit the high flux of atmospheric neutrinos at energies of a few hundred $\mathrm{GeV}$ to observe a set of 1,029 cascade-like neutrino events in 281 days of the 2010 data run. One such event is shown in Fig. 4

For this data set, recorded with the incomplete 79string configuration of IceCube, the smaller DeepCore fiducial volume shown in Fig. 1 was used. This initial configuration consisted of only the central seven standard strings, plus 6 additional DeepCore strings. Based on Monte Carlo simulations, we estimate that approximately $60 \%$ of the 1,029 events in the final sample are truly neutrino-induced cascades, while around $40 \%$ are in fact $v_{\mu}$ CC events with muon tracks too short to be distinguished in the current analysis; efforts to further reduce this background are underway. The level of background due to atmospheric muons is still under investigation but appears to be small. The rates of observed neutrinos are consistent with simulations of at- 


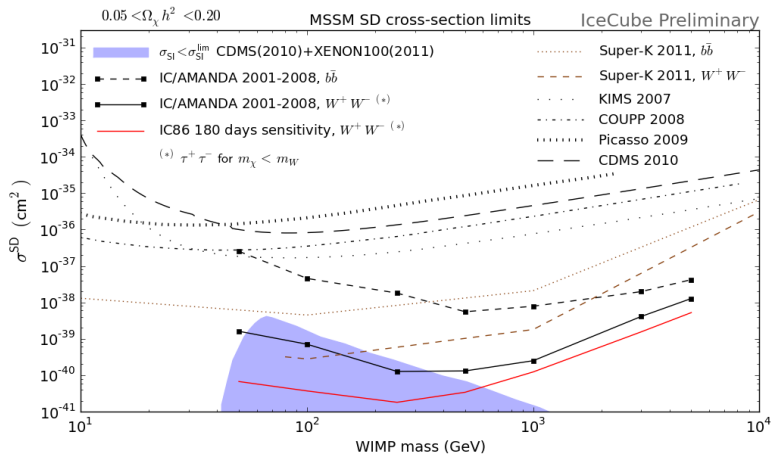

Figure 5: Limits on the spin-dependent WIMP-nucleon scattering cross section from various direct and indirect search experiments, and the projected sensitivity of IceCube with DeepCore for a "hard" neutrino spectrum arising from neutralino annihilation in the Sun. The shaded region indicates the possible cross sections in supersymmetric models not already ruled out by direct detection experiments' limits on the spin-independent cross section.

mospheric neutrinos using the leading atmospheric neutrino flux models from the Bartol and Honda groups, although we are still in the process of assessing our systematic uncertainties. It should be noted that the predictions based on the two atmospheric flux models differ for this event set by approximately $10 \%$, due mainly to the modeling of production of higher energy electron neutrinos by kaons.

Work is in progress to lower the energy threshold of the analysis, which would permit observation of neutrino oscillations using the atmospheric neutrino flux. For baselines comparable to the Earth's diameter, the first maximum of the $v_{\mu} \rightarrow v_{\tau}$ oscillation probability occurs at approximately $25 \mathrm{GeV}$, well within the energy range accessible to DeepCore [7].

\section{Searches for Dark Matter}

In addition to studies of atmospheric neutrinos, DeepCore's reduced energy threshold facilitates indirect searches for evidence of dark matter using IceCube. Searches are underway for neutrinos produced in the annihilation or decay or dark matter captured in the gravitational potential wells of the Earth, Sun [8, 9], and Galaxy [10]. Because the WIMP mass must be relatively low compared to the energy range of IceCube, additional sensitivity to lower energy neutrinos substantially extends IceCube's reach, especially for the lower part of the allowed WIMP mass range or for models where the neutrino spectrum produced is relatively soft.

The potential of IceCube including DeepCore for detecting evidence of dark matter annihilation in the
Sun is shown in Fig. 5. The shaded region indicates the allowed MSSM parameter space, for models where the WIMP is a neutralino. Direct detection experiments have already probed substantial parts of the allowed supersymmetric parameter space, primarily in regions where there is a substantial spin-independent neutralino-nucleon scattering cross section, so that coherent scattering from heavy nuclei in the detector target enhances the cross section considerably. For models in which the scattering cross section is primarily spindependent, indirect searches exploiting the Sun's mass as a scattering target have an advantage, although the results depend on the branching ratios for neutralinoneutralino annihilation channels. For WIMP masses below roughly $100 \mathrm{GeV}$, DeepCore provides the bulk of the sensitivity to the neutrinos arising from Solar neutralino annihilation.

\section{Future Prospects: PINGU}

Encouraged by the initial success of DeepCore, the IceCube collaboration and other participants are developing a proposal for a Phased IceCube Next Generation Upgrade (PINGU), an extension of IceCube and DeepCore which would further increase the density of instrumentation in the central volume and further reduce the energy threshold. The proposal would augment DeepCore with perhaps 18 to 20 additional strings, of which the majority would be similar to those in DeepCore. Several strings might also include specialized prototypes of novel sensors, perhaps similar to those incorporating a number of 3" PMTs rather than a single 10" PMT, now being developed for the proposed KM3NeT detector. One layout of the additional strings under discussion is shown in Fig. 6

Such an extension would considerably increase the effective volume of DeepCore at energies below about $30 \mathrm{GeV}$, with the potential to detect neutrinos as low as a few $\mathrm{GeV}$. The effective volume of the detector for events contained within the geometrical volume is shown in Fig. 7, as compared with that of the existing DeepCore detector. Improvements of nearly an order of magnitude can be seen for low energy neutrinos. These effective volumes do not include efficiency losses due to event reconstruction and analysis criteria, which will reduce the effective volume achievable in final physics analysis.

\section{Summary}

The effectiveness of IceCube at energies below 100 $\mathrm{GeV}$ has been significantly enhanced by the addition 


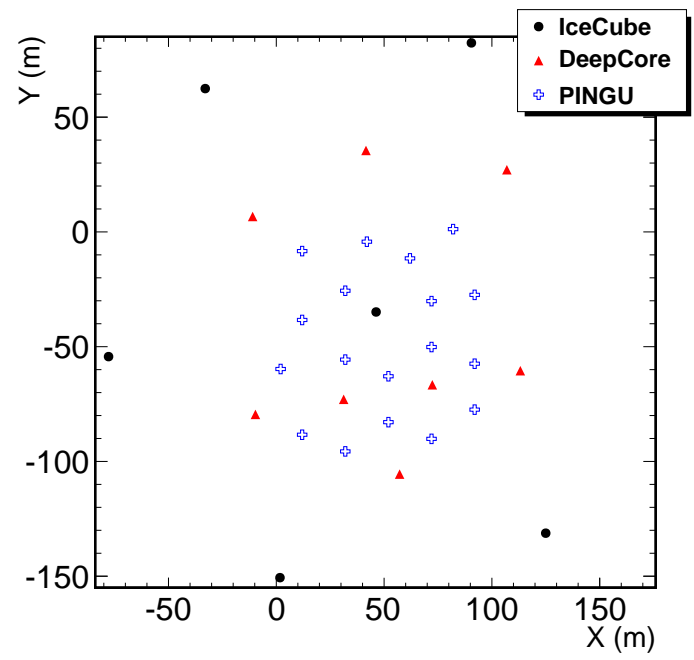

Figure 6: Top view of one PINGU configuration now under study, including 16 strings of DeepCore-like instrumentation. Additional strings of prototype next-generation instrumentation are envisioned but not shown. This layout would significantly improve the effectiveness of DeepCore at energies below a few 10's of GeV.

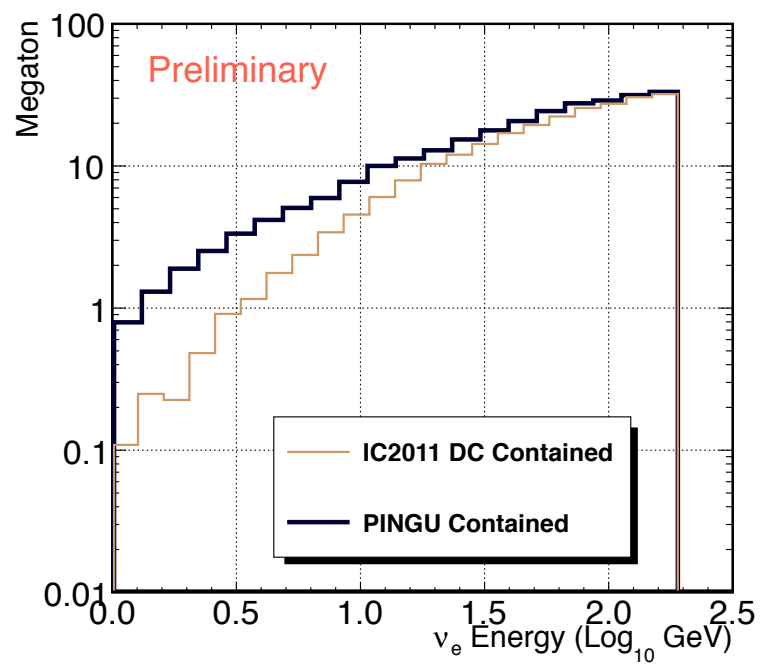

Figure 7: Preliminary estimate of the effective volume of PINGU for electron neutrino events at trigger level, as compared to that of the completed DeepCore configuration. PINGU would retain considerable effective volume down to energies as low as a few GeV. Analysis and reconstruction efficiencies are not included. The geometry used for this estimate is similar to that shown in Figure 6 but with a slightly larger mean spacing between strings, so the effective volume at the lowest energies may be underestimated. of DeepCore, which extends IceCube's reach to energies of 10's of GeV. This range is of interest for observations of neutrino oscillations, as well as searches for dark matter. As a first step toward these studies, we have observed a significant sample of atmospheric neutrinoinduced cascades, enabled by the ability of the IceCube detector to identify and veto atmospheric muons penetrating to the DeepCore volume. We are also investigating the potential for a further reduction in the energy threshold of IceCube with an additional extension known as PINGU, which could extend IceCube's reach to energies as low as a few $\mathrm{GeV}$

\section{References}

\section{References}

[1] R. Abbasi et al. [The IceCube Collaboration], arXiv:1109.6096 [astro-ph.IM].

[2] J. Ahrens et al. [The AMANDA Collaboration], Phys. Rev. D 67, 012003 (2003). arXiv:astro-ph/0206487].

[3] M. Ackermann et al. [The AMANDA Collaboration], Astropart. Phys. 22, 127 (2004). [arXiv:astro-ph/0405218].

[4] A. Achterberg et al. [The IceCube Collaboration], Astrophys. J. 664, 397 (2007). |arXiv:astro-ph/0702265].

[5] R. Abbasi et al. [The IceCube Collaboration], Astropart. Phys. 34, 420-430 (2011).

[6] R. Abbasi et al. [The IceCube Collaboration], Phys. Rev. D 84, 072001 (2011). arXiv:1101.1692 [astro-ph.HE]]

[7] O. Mena, I. Mocioiu and S. Razzaque, Phys. Rev. D 78, 093003 (2008). [arXiv:0803.3044 [hep-ph]].

[8] R. Abbasi et al. [The IceCube Collaboration ], Phys. Rev. Lett. 102, 201302 (2009).

[9] R. Abbasi et al. [The IceCube Collaboration], Phys. Rev. D81, 057101 (2010).

[10] R. Abbasi et al. [The IceCube Collaboration], Phys. Rev. D84, 022004 (2011). [arXiv:1101.3349 [astro-ph.HE]]. 